\title{
Eliciting vulnerable patients' preferences regarding colorectal cancer screening: a systematic review
}

This article was published in the following Dove Press journal:

Patient Preference and Adherence

\author{
Samuel J Lee' \\ Meghan C O'Leary' \\ Karl E Umble \\ Stephanie B Wheeler ${ }^{1-3}$ \\ 'Department of Health Policy and \\ Management, University of North \\ Carolina at Chapel Hill, Chapel Hill, \\ NC, USA; ${ }^{2}$ Lineberger Comprehensive \\ Cancer Center, University of North \\ Carolina at Chapel Hill, Chapel \\ Hill, NC, USA; ${ }^{3}$ Center for Health \\ Promotion and Disease Prevention, \\ University of North Carolina at \\ Chapel Hill, Chapel Hill, NC, USA
}

Background: Patient preferences are important to consider in the decision-making process for colorectal cancer (CRC) screening. Vulnerable populations, such as racial/ethnic minorities and low-income, veteran, and rural populations, exhibit lower screening uptake. This systematic review summarizes the existing literature on vulnerable patient populations' preferences regarding CRC screening.

Methods: We searched the CINAHL, PsycINFO, PubMed, Scopus, and Web of Science databases for articles published between January 1, 1996 and December 31, 2017. We screened studies for eligibility and systematically abstracted and compared study designs and outcomes. Results: A total of 43 articles met the inclusion criteria, out of 2,106 articles found in our search. These 43 articles were organized by the primary sub-population(s) whose preferences were reported: 27 report on preferences among racial/ethnic minorities, eight among low-income groups, six among veterans, and two among rural populations. The majority of studies $(n=34)$ focused on preferences related to test modality. No single test modality was overwhelmingly supported by all sub-populations, although veterans seemed to prefer colonoscopy. Test attributes such as accuracy, sensitivity, cost, and convenience were also noted as important features. Furthermore, a preference for shared decision-making between vulnerable patients and providers was found.

Conclusion: The heterogeneity in study design, populations, and outcomes of the selected studies revealed a wide spectrum of CRC screening preferences within vulnerable populations. More decision aids and discrete choice experiments that focus on vulnerable populations are needed to gain a more nuanced understanding of how vulnerable populations weigh particular features of screening methods. Improved CRC screening rates may be achieved through the alignment of vulnerable populations' preferences with screening program design and provider practices. Collaborative decision-making between providers and vulnerable patients in preventive care decisions may also be important.

Keywords: colorectal cancer screening, systematic review, vulnerable populations, patient preference

\section{Introduction}

Colorectal cancer (CRC) is the third most common type of cancer and the second leading cause of cancer deaths in the US. In 2017, there were an estimated 135,430 new cases diagnosed and 50,260 CRC-specific deaths nationally. ${ }^{1}$ Annually, CRC costs the US healthcare system approximately $\$ 14$ billion. ${ }^{2}$ Screening has been shown to reduce CRC incidence and mortality by $30 \%-60 \%$ and has the potential to save an estimated 18,800 lives per year. ${ }^{3,4}$ Since early stage CRC is asymptomatic, screening is especially important for early detection and appropriate treatment. ${ }^{5}$ The US Preventive Services Task Force (USPSTF) has recommended screening using colonoscopy, sigmoidoscopy,
Correspondence: Stephanie B Wheeler Department of Health Policy and Management, University of North Carolina at Chapel Hill, I I 04E McGavranGreenberg Hall CB \#74I, I Chapel Hill, NC 27599-74II, USA

Tel + I 9199667374

Email stephanie_wheeler@unc.edu (c) (1) (5) 2018 Lee et al. This work is published and licensed by Dove Medical Press Limited. The full terms of this license are available at https://www.dovepress.com/terms.php (c) hereby accept the Terms. Non-commercial uses of the work are permitted without any further permission from Dove Medical Press Limited, provided the work is properly attributed. For permission for commercial use of this work, please see paragraphs 4.2 and 5 of our Terms (https://www.dovepress.com/terms.php). 
or fecal testing, such as fecal occult blood testing (FOBT) and fecal immunochemical testing (FIT), at appropriate intervals (eg, colonoscopy every 10 years, sigmoidoscopy every 5 years, fecal testing annually), for average-risk adults aged 50-75 years. ${ }^{6}$ Colonoscopy and fecal testing are the most commonly used modalities in the US. ${ }^{7}$

The US has seen an increase in CRC screening over time, yet the 2015 national rate of $62.6 \%$ is well below the Healthy People 2020 target of $70.5 \%$ set by the US Department of Health and Human Services. ${ }^{8,9}$ CRC screening rates are particularly low within many vulnerable sub-populations, including racial and ethnic minorities and foreign-born, low-income, publicly insured, uninsured, veteran, disabled, lesbian, gay, bisexual, transgender, queer (LGBTQ), and rural populations. ${ }^{10-15}$ Lack of consideration of patient preferences is one of several factors contributing to lower screening rates. ${ }^{16}$ To improve uptake, communication between providers and patients about decision alternatives, preferences, and risk-benefit tradeoffs is important. ${ }^{17}$ Shared decision-making, in which the provider and patient work together to agree upon an optimal decision, has been increasingly recommended for screening. ${ }^{18}$ Considerations for $\mathrm{CRC}$ screening include test characteristics, such as accuracy, invasiveness, and comfort, and delivery characteristics, including cost, convenience, and ease of access. ${ }^{19}$ For example, some patients may prefer fecal testing, due to its noninvasive nature and low cost. Presenting choices that match individuals' preferences may increase CRC screening uptake.
Systematic reviews have previously assessed CRC screening preferences within the general population and found that while accuracy and clinical effectiveness are valued, there is not an overwhelming preference for a single modality. ${ }^{20-22}$ Given that vulnerable populations screen at lower rates and face greater barriers to preventive care, their CRC screening preferences may differ in important ways. ${ }^{23-27}$ Thus, a better understanding of preferences among vulnerable populations is needed to inform interventions and policies that aim to increase their CRC screening rates. To do this, we conducted a systematic review with the objective of capturing the preferences of vulnerable populations with respect to CRC screening. We aimed to capture all aspects of preference in the context of screening, such as modality, test attributes, and program features. To our knowledge, this is the first systematic review to address CRC screening preferences specifically among vulnerable patients.

\section{Methods}

We conducted a systematic review according to the Preferred Reporting Items for Systematic Reviews and Meta-Analyses guidelines. ${ }^{28} \mathrm{We}$ adapted the Population, Intervention, Comparison, Outcome, Time (PICOT) framework, adding in both study setting and design, to identify the studies of interest in this review. ${ }^{29}$ Since the PICOT framework is often used in the context of eliciting the effect of a treatment, and we were interested in reviewing a wide range of study types, some of which did not include a specific intervention, we omitted the intervention component of the framework. Table 1 outlines

Table I Inclusion/exclusion criteria

\begin{tabular}{|c|c|c|}
\hline Criterion & Inclusion & Exclusion \\
\hline Population & $\begin{array}{l}\text { Vulnerable patient populations } \\
\text { - Rural residents } \\
\text { - Racial/ethnic minorities } \\
\text { - Low-income populations } \\
\text { - Limited English proficiency (LEP) or non-English speaking } \\
\text { - Immigrants/foreign-born } \\
\text { - Disabled } \\
\text { - Lesbian, gay, bisexual, transgender, queer (LGBTQ) populations } \\
\text { - Medicaid enrollees } \\
\text { - Dually insured (Medicare/Medicaid) } \\
\text { - Uninsured } \\
\text { - Veterans }\end{array}$ & $\begin{array}{l}\text { All other non-vulnerable } \\
\text { patient populations }\end{array}$ \\
\hline Comparison & Modalities of CRC screening as well as screening program delivery features and other attributes & All other comparisons \\
\hline Outcome & $\begin{array}{l}\text { Patient-level CRC screening preferences related to test modalities, test features, incentives, } \\
\text { screening program design, service delivery, source of information, communication method }\end{array}$ & All other outcomes \\
\hline Time & Articles published from January I, 1996 to December 3I, 2017 & $\begin{array}{l}\text { Articles published outside } \\
\text { of this time period }\end{array}$ \\
\hline Setting & All developed country settings (including international studies) & Developing countries \\
\hline Study design & $\begin{array}{l}\text { Quantitative (including discrete choice experiments/conjoint analyses), qualitative, and } \\
\text { mixed-methods }\end{array}$ & $\begin{array}{l}\text { Literature reviews, } \\
\text { systematic reviews }\end{array}$ \\
\hline
\end{tabular}


the inclusion and exclusion criteria for this review based on the adapted PICOT framework.

We focus our review on vulnerable populations who experience widely observed health disparities and are at risk for poor quality of care and poor health outcomes due to non-clinical, discriminatory, and marginalizing factors. ${ }^{30-34}$ The National Academy of Medicine (formerly Institute of Medicine) and Agency for Healthcare Research and Quality have, for decades, placed elimination of health disparities at the center of healthcare quality initiatives, noting that highquality care should not be differentially received by people because of race, ethnicity, gender identity, sexual preference, geography, or socioeconomic status. ${ }^{35-38}$ The US Department of Health and Human Services (HHS) Office of Minority Health describes a health disparity as "a particular type of health difference that is closely linked with social, economic, and/or environmental disadvantage." ${ }^{30,39}$ HHS characterizes underserved, vulnerable, and special need populations as communities that include members of minority populations or individuals who have experienced health disparities, specifically Latinos, African Americans, American Indians/ Alaska Natives, refugees/migrants, individuals with Limited English Proficiency (LEP), uninsured, low-income, rural, LGBT, and disabled people, including disabled veterans, as well as pregnant women and children. ${ }^{39,40} \mathrm{We}$ adopt this inclusive definition of "vulnerable and medically underserved" populations in our review, excluding pregnant women and children, who are not age-eligible for CRC screening and therefore not relevant to this review.

Since little was known about the literature addressing vulnerable patients' CRC screening preferences, including the types of preferences assessed, we attempted to cast a wide net on this topic. We included all articles that met our review criteria with a variety of study designs (eg, observational and experimental) because our goal was to understand vulnerable patients' preferences about CRC screening generally. That is to say, we did not constrain our review to specific aspects of preferences, such as preference tradeoffs or changes in preference as a result of experimental intervention; both of these types of articles were viewed as relevant and within scope.

We searched the PubMed, CINAHL, PsycINFO, Scopus, and Web of Science databases for articles published from January 1, 1996 to December 31, 2017 (Figure 1). We selected this timeframe because the USPSTF released its first CRC screening guidelines in December 1995. ${ }^{41}$ The following search string was used to identify relevant articles: ((“colorectal” AND "cancer" OR “colon" AND "cancer") AND ("screening” OR “detection" OR "testing” OR "test") AND (("preference” OR “preferences" OR "perception” OR "perceptions") OR ("discrete" AND "choice") OR (discrete AND choices))) AND PUBYEAR > 1995.

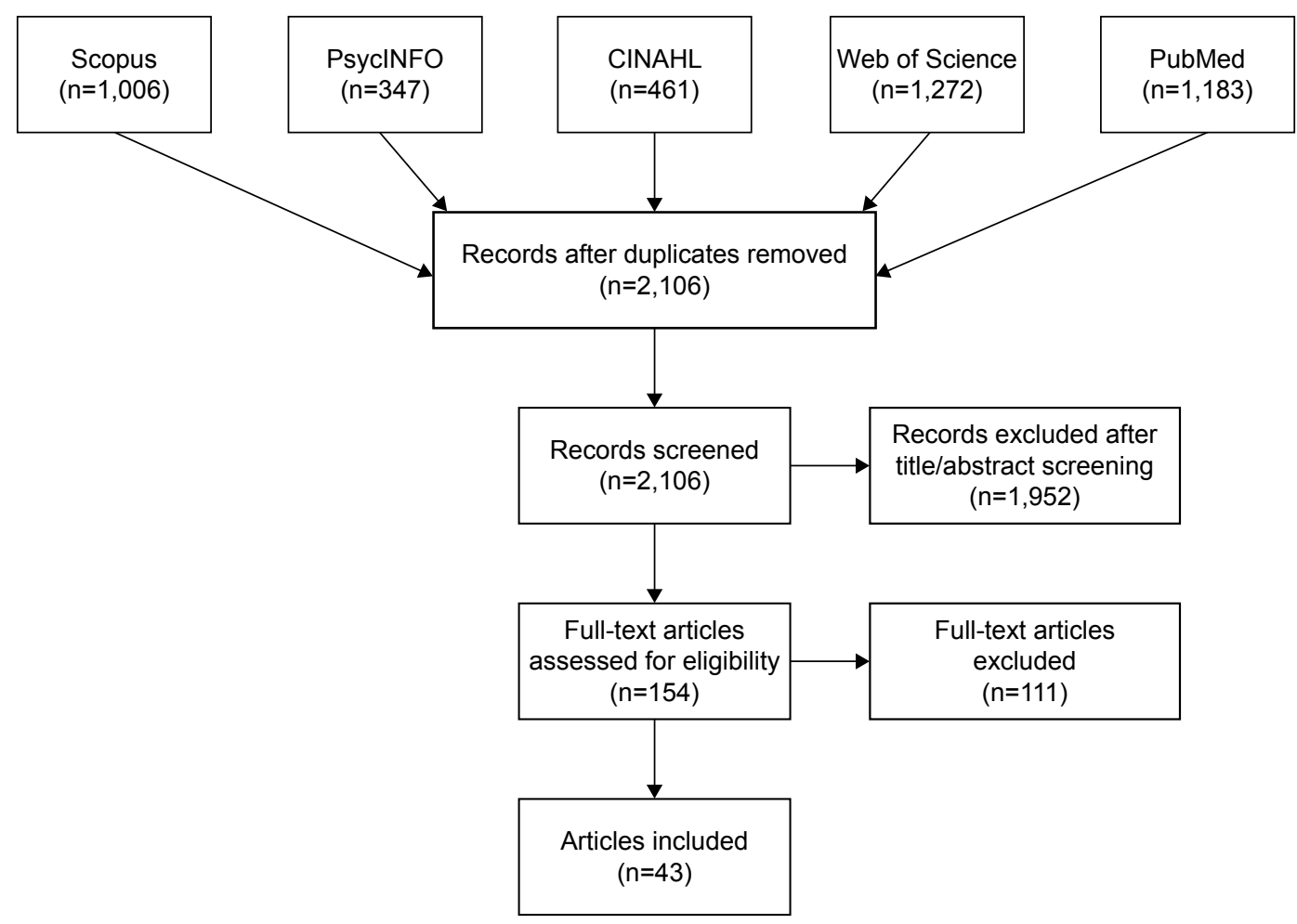

Figure I PRISMA Flow Diagram. 
Since our goal was to capture a range of vulnerable populations, we did not list the particular populations of interest, as identified in Table 1, in this initial search string. This strategy allowed us to discern whether vulnerable populations were included in each article rather than exclude studies outright that failed to mention a specific term that we associated with "vulnerable" or "underserved." Studies from the US and other developed country contexts that comprised subgroup analyses regarding screening preferences for one or more vulnerable populations were eligible for inclusion.

In total, 4,269 articles were initially identified and imported into F1000 Workspace (Faculty of 1000 Ltd, 2018), a reference management database. After duplicates were removed, 2,106 articles were transferred to Covidence (Veritas Health Innovation Ltd, 2018) to be screened.

Using Covidence, two reviewers ( $\mathrm{SL}$ and $\mathrm{MO}$ ) independently screened the titles and abstracts of the articles for patient-level studies focused on CRC screening. The reviewers began with a small pool of 20 articles to ensure consistency and to refine the inclusion/exclusion criteria. These reviewers resolved any discrepancies in their ratings and, when a consensus could not be reached, a third reviewer (SW) assessed the article and made the final decision. These same procedures were then used to review the titles and abstracts of the remaining articles. In this initial phase, we excluded articles that were not related to CRC screening, such as studies about other health conditions, CRC studies regarding treatment, survivorship, or genetics, and nonpatient-level CRC studies (eg, provider-only interventions). This strategy provided the opportunity to review the full-text of CRC screening studies to consider if a sub-population analysis was included and if a preference-related outcome was measured, even if the larger objective of the article was not specific to these areas. During the title/abstract screening process, 1,952 articles were removed, leaving 154 full-text articles to be reviewed.

During the full-text review, the reviewers assessed whether each article should be included or excluded according to the criteria outlined in Table 1 and categorized the excluded articles by reason for exclusion. Discrepancies regarding whether the article should be included or excluded, as well as the reason for exclusion, were resolved by the two reviewers (SL and MO) with the third reviewer (SW) making the final decision about any remaining discrepancies. Of the 154 full-text articles, 111 were excluded, most commonly because they did not provide a subgroup analysis for a vulnerable population $(\mathrm{n}=70)$. The other primary reasons for exclusion were outcomes outside the scope of this analysis, such as the reporting of screening behaviors without addressing patient preferences $(n=20)$, the wrong study design including systematic and literature reviews $(\mathrm{n}=16)$, duplicate studies ( $\mathrm{n}=3$ ), non-English publications $(\mathrm{n}=1)$, and publication dates outside the study window $(n=1)$.

The two reviewers abstracted the data from the included articles into a literature matrix using Microsoft Excel 2010 (Microsoft Corporation, Redmond, WA). The literature matrix included more than 20 structured fields such as title, study design, US or international study, sub-population studied, total sample size and the sample size of the vulnerable patients, baseline population characteristics (eg, age, sex, race), outcome measure, and findings. For the studies in which an intervention was implemented, we also reported the type of intervention, mode of delivery, and who conducted the intervention. Given the number of metrics and heterogeneity across studies, in this paper we report the primary type of vulnerable population included in each article, study objective, study design, sample sizes of the total and vulnerable populations, outcome measured, and findings to provide an understanding of the breadth of research currently available on vulnerable patients' preferences.

\section{Results}

A total of 43 articles that addressed patient preferences regarding CRC screening among vulnerable groups are included in this systematic review. The selected articles are organized by the types of vulnerable population(s) whose preferences are reported. Of these studies, 27 reported preferences among racial/ethnic minorities, eight among low-income groups, six among veterans, and two among rural populations. Notably, many studies elicited preferences from more than one underserved population, since these categories are not necessarily mutually exclusive. For example, studies often included racial or ethnic minorities as well as low-income individuals. In this analysis, studies were categorized based on the vulnerable population prioritized during sampling and analysis.

Grouping by outcomes or study design, rather than subpopulation of interest, was considered, but we ultimately decided that categorizing by sub-population would better assist public health practitioners and researchers in designing interventions for these specific sub-groups. However, within each section, we organized the study results by outcome. Of the 43 studies, 34 measured preference in terms of modality, displaying a marked tendency to focus on modality rather than other aspects of preference within research; 23 measured preference in terms of test attributes. The other types of 
preferences assessed were source of information ( $\mathrm{n}=2)$, type of decision-making $(n=2)$, program delivery $(n=2)$, expert recommendation $(\mathrm{n}=1)$, willingness to pay (WTP; $\mathrm{n}=1$ ), and provider characteristics $(\mathrm{n}=1)$.

\section{Racial/ethnic minorities $(\mathrm{n}=27)$}

Twenty-seven articles reported CRC screening preferences for a single or multiple racial/ethnic minority groups (Table 2). ${ }^{42-68}$ Out of these studies, nine were conducted among African Americans, ${ }^{42,44,48,49,54,57-59,61}$ five among Hispanics/Latinos, ${ }^{47,53,55,62,68}$ eight among both African Americans and Hispanics/Latinos, ${ }^{43,45,50,51,63-66}$ four among non-whites, ${ }^{46,56,60,67}$ and one among Korean Americans. ${ }^{52}$ Race was self-reported in nearly all of these articles, except for a study in which recruitment was conducted at a communitybased organization serving Korean Americans, ${ }^{52}$ a study that used health insurance claims data with enrollment database-reported race/ethnicity, ${ }^{59}$ and a study that did not clearly specify how race/ethnicity was obtained..$^{63}$ In terms of outcomes, 22 studies reported preferences in terms of test modality and/or specific test attributes. ${ }^{42-46,48-51,53,56-67}$ Of the remaining five articles, two examined preferences regarding shared decision-making, ${ }^{54,55}$ two investigated preferred sources of information, ${ }^{47,52}$ and one considered preference for the sex and ethnicity of endoscopists. ${ }^{68}$

\section{Screening modality}

There was a significant variation in the preferred screening modality within the studies primarily focused on minority racial and ethnic groups. Five papers reported colonoscopy as the preferred test, six studies reported fecal testing as the preferred screening method, four studies found mixed or inconclusive results, and five studies found that race/ethnicity was not associated with preferred modality. Of those who reported colonoscopy as the preferred test, four focused on African Americans only, ${ }_{48,57,58,61}$ and one included both African Americans and Hispanics. ${ }^{45}$ Of the six studies that reported fecal testing as the preferred screening method, two studies reported a preference for home-based fecal tests over colonoscopy among multiracial/multiethnic groups ${ }^{43,67}$ one study reported a preference specifically for FOBT among minority racial groups, ${ }^{46}$ one study reported a preference specifically for FIT among African Americans, ${ }^{49}$ and two studies, one among African Americans ${ }^{42}$ and one among a non-white study population ${ }^{60}$ identified stool-based DNA testing as the preferred option. Finally, of the five studies that found that race/ethnicity was not associated with preferred test modalities, four found colonoscopy as the preferred screening test in the general population; ${ }^{44,50,56,59}$ the remaining study found that FOBT was preferred over colonoscopy in the general population. ${ }^{66}$

\section{Test attributes}

Nine articles reported attributes that racial and ethnic minority patients value in particular CRC screening tests or programs. ${ }^{42,45,51,53,57,61,63,65,66}$ Test accuracy was commonly reported as an important attribute, regardless of the preferred test or particular group. ${ }^{42,45,51,57,61,63}$ For example, although Hawley et al found differences in the preferred modality between African Americans, Hispanics, and whites, accuracy was rated as the most important attribute across all groups. ${ }^{51}$ Accuracy was also an influential factor among African Americans who preferred sDNA, ${ }^{42}$ as well as African Americans who preferred colonoscopy. ${ }^{61}$ Palmer et al reported that accuracy and thoroughness were the most positive attributes of colonoscopy, while test ease and noninvasiveness were viewed as the best attributes of FOBT for African Americans. ${ }^{57}$ Similarly, Chablani et al found that the most preferred attribute of colonoscopy is test accuracy, whereas the top attribute of Cologuard is the lack of preparation needed, in a sample of African Americans and Hispanics. ${ }^{45}$ Among African Americans, Hispanics/Latinos, and whites who prefer FOBT, convenience was identified as the most important attribute. ${ }^{66} \mathrm{~A}$ discrete choice experiment conducted among Latinos determined that patients in the study were more concerned about the costs of screening and any required follow-up care than the type of modality used or the amount of time required for travel. ${ }^{52}$

This review also highlighted some differences in perceptions regarding test attributes between racial and ethnic groups. Although African Americans and Hispanics/Latinos both ranked accuracy and comprehensiveness of the test as two of the most important features, African Americans were also concerned about discomfort and potential complications, while Hispanics/Latinos focused on the extent of scientific evidence available. ${ }^{63}$ African Americans were more likely to be embarrassed by stool-based DNA testing than whites, ${ }^{42}$ and less likely to associate low cost with SEPT9 blood testing, and to consider the frequency of each test as important to decision-making, compared to whites and Hispanics/Latinos. ${ }^{51,63}$

\section{Source of information}

Two articles addressed minority patients' preferences for their source of information regarding CRC screening. ${ }^{47,52}$ Jo et al conducted interviews among Korean Americans and 


\begin{tabular}{|c|c|c|c|c|c|c|c|}
\hline 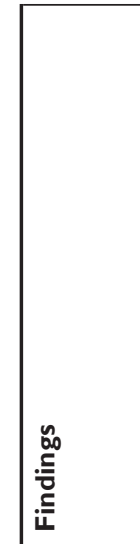 & 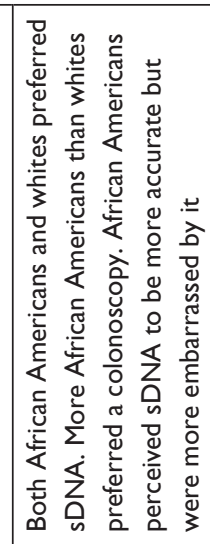 & 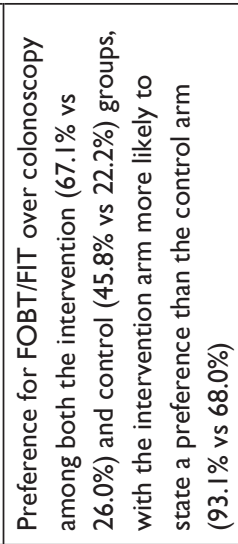 & 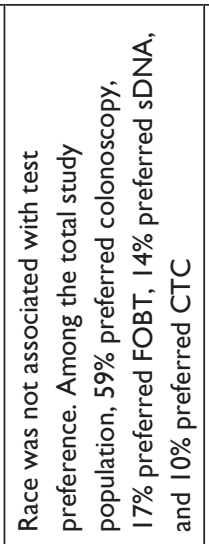 & 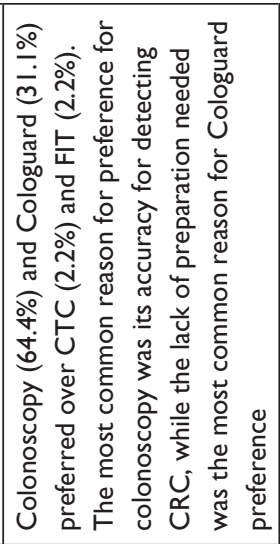 & 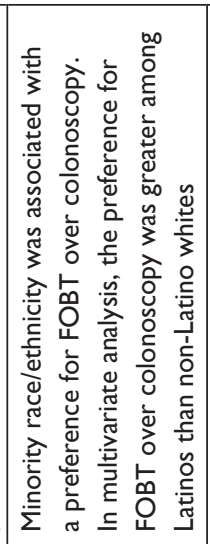 & 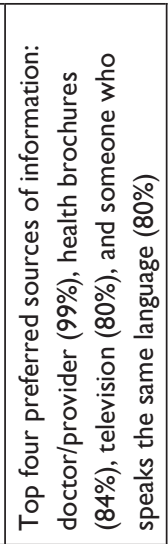 & 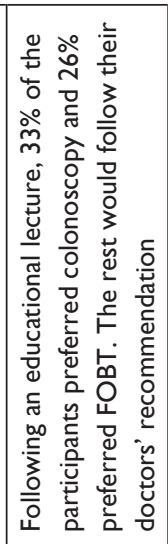 \\
\hline 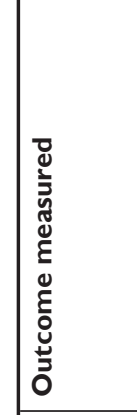 & 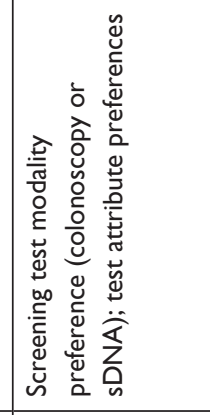 & 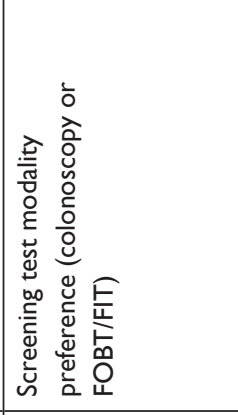 & 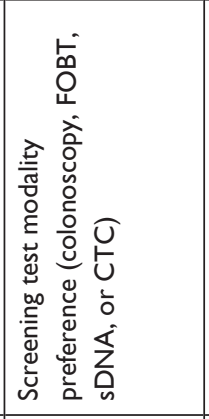 & 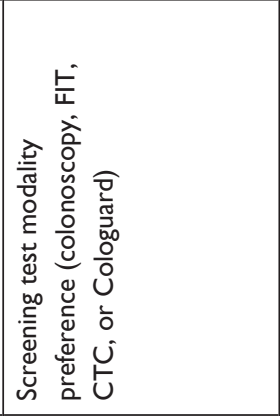 & 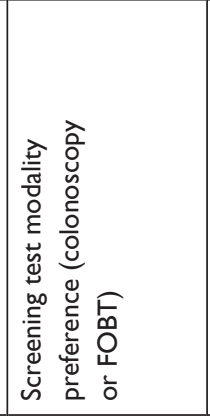 & 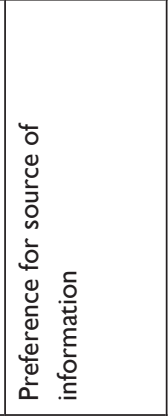 & 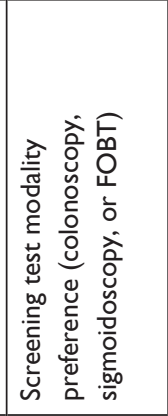 \\
\hline \begin{tabular}{|ll} 
\\
\\
4
\end{tabular} & $\underline{\Xi}$ & ปั & $\stackrel{m}{n}$ & ঃ & 点 & 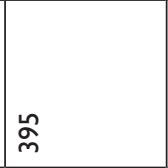 & 饮 \\
\hline 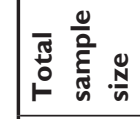 & $\widetilde{q}$ & స్ & 음 & 2 & $\stackrel{\tilde{m}}{.}$ & 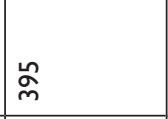 & 㶽 \\
\hline 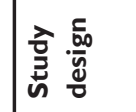 & 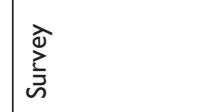 & $\begin{array}{l}\qquad \\
\check{x}\end{array}$ & 离 & 离 & 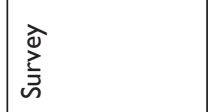 & 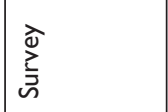 & 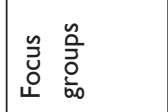 \\
\hline 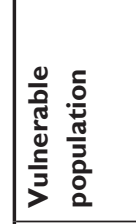 & 悹 & 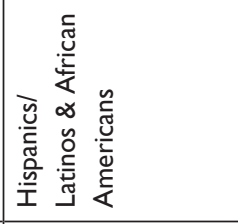 & 竞 & 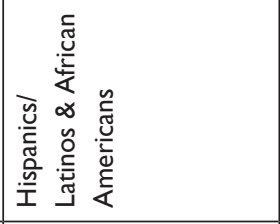 & 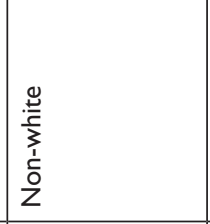 & 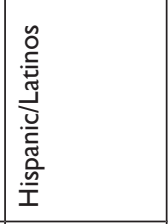 & 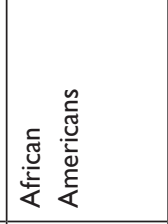 \\
\hline 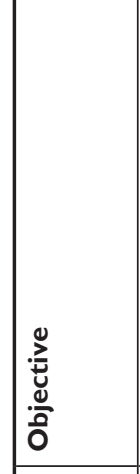 & 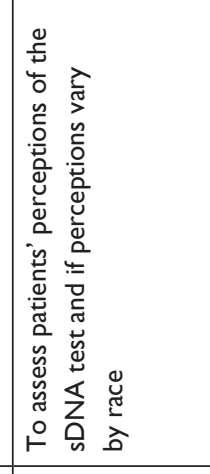 & 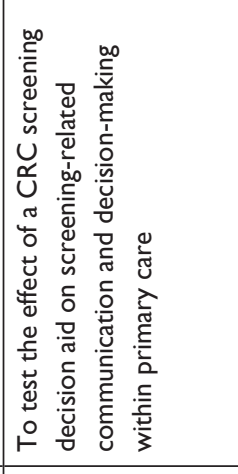 & 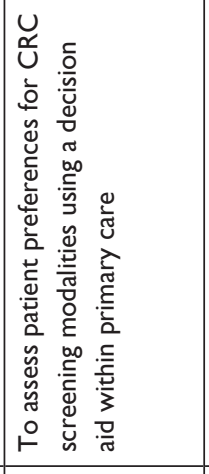 & 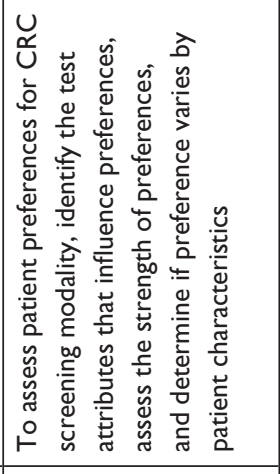 & 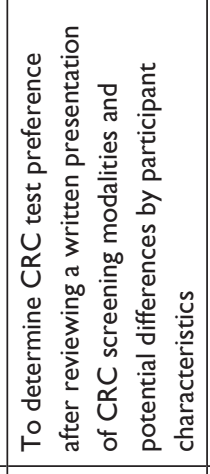 & 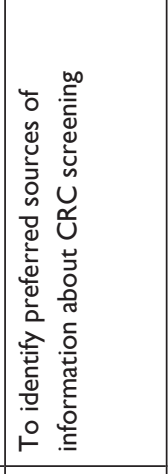 & 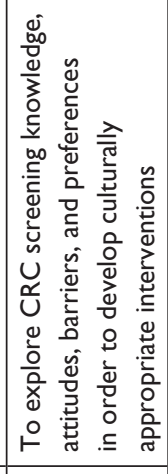 \\
\hline $\begin{array}{l}\text { ते } \\
\text { 竞 }\end{array}$ & 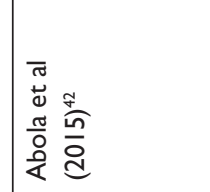 & 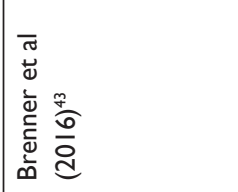 & 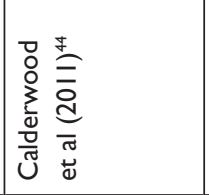 & 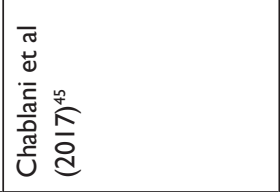 & 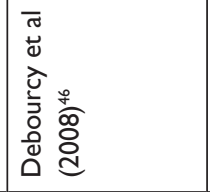 & 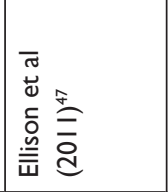 & 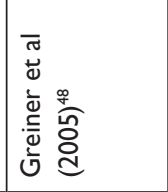 \\
\hline
\end{tabular}




\begin{tabular}{|c|c|c|c|c|c|c|}
\hline 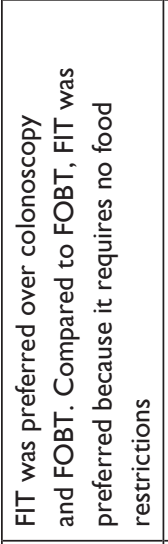 & 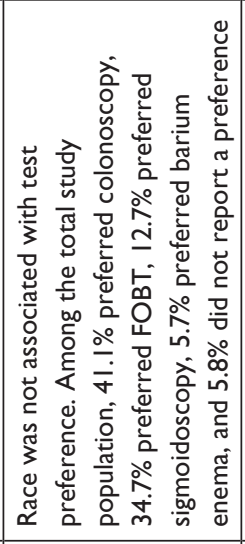 & 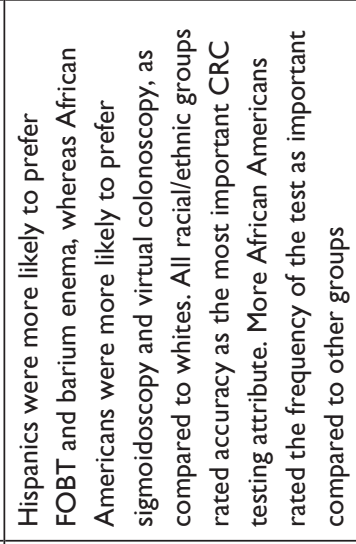 & 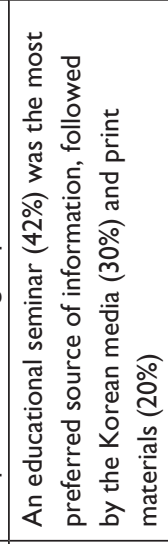 & 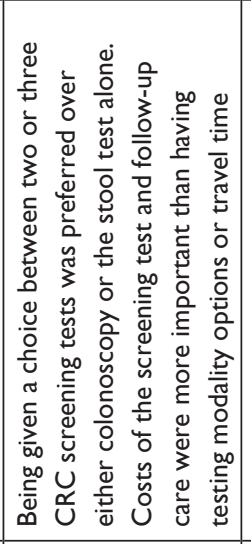 & 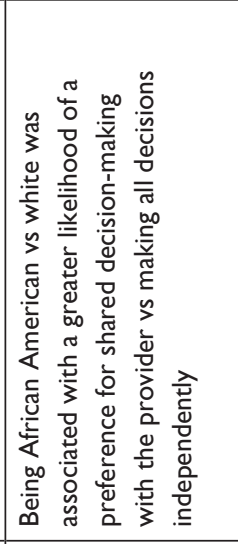 & 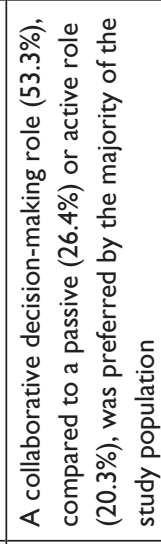 \\
\hline 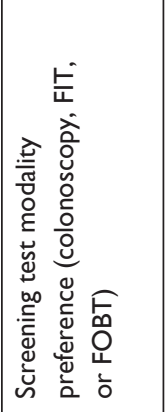 & 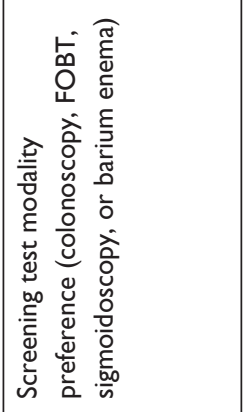 & 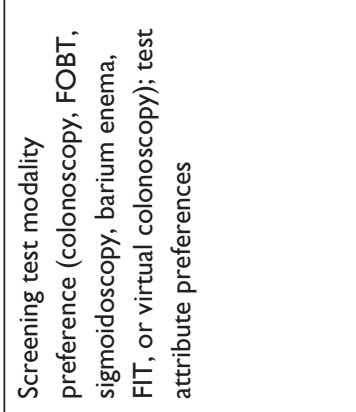 & 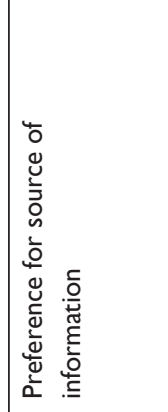 & 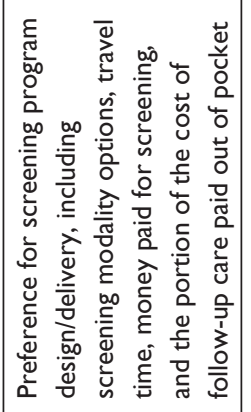 & 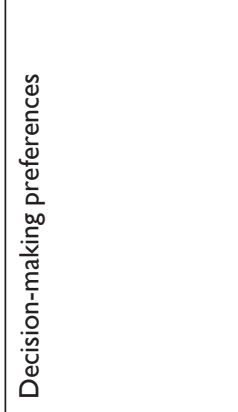 & 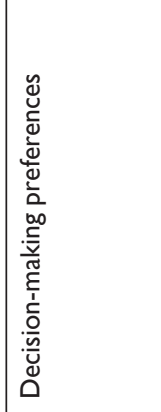 \\
\hline$\stackrel{\infty}{\sim}$ & $\stackrel{\circ}{2}$ & 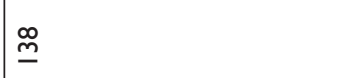 & $\underline{\underline{n}}$ & $\underset{m}{\infty}$ & $\underline{\Xi}$ & $\stackrel{\otimes}{\stackrel{\circ}{\prime}}$ \\
\hline$\stackrel{\infty}{\sim}$ & 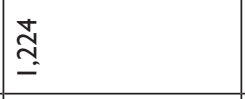 & $\stackrel{\sim}{N}$ & $\underline{\text { ㅁ}}$ & $\stackrel{\infty}{m}$ & $\frac{\sigma}{i}$ & $\stackrel{\infty}{\propto}$ \\
\hline 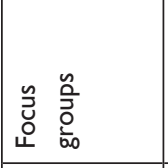 & 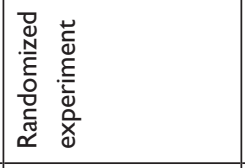 & 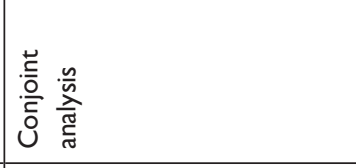 & 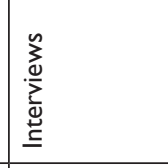 & 岂 & 离 & 俞 \\
\hline 胥 & 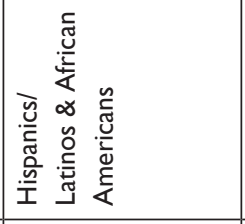 & 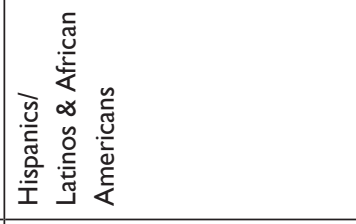 & 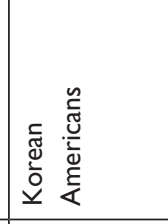 & 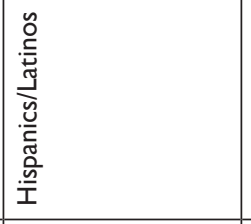 & 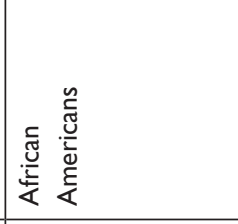 & 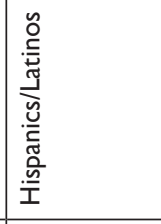 \\
\hline 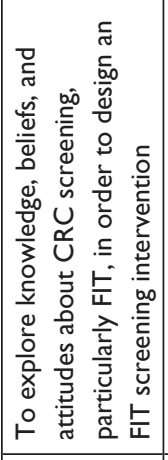 & 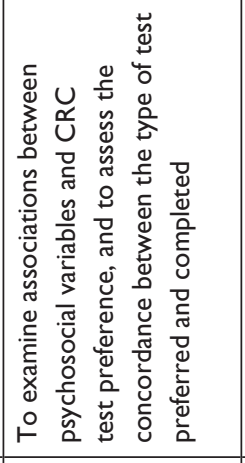 & 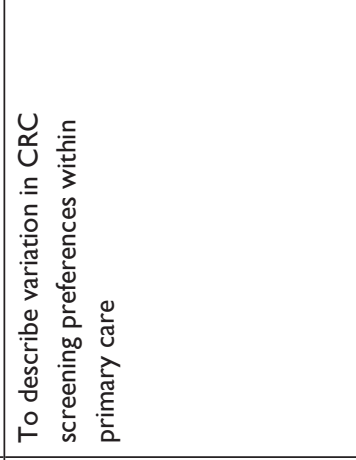 & 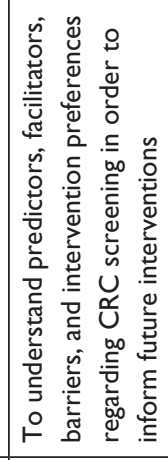 & 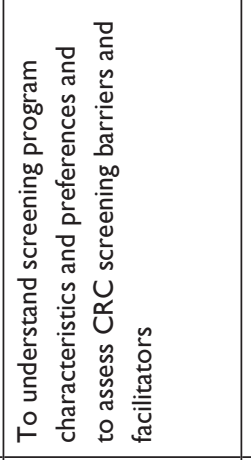 & 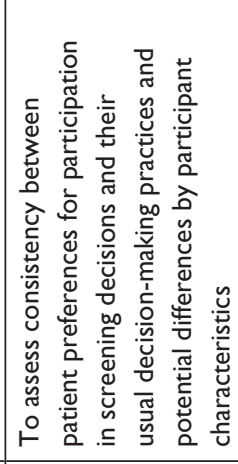 & 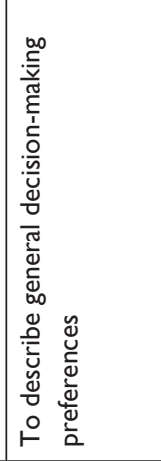 \\
\hline 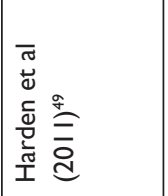 & 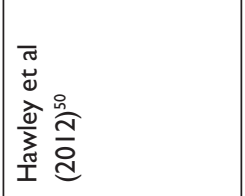 & 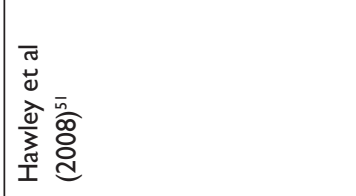 & 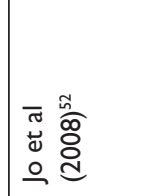 & 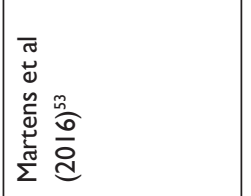 & 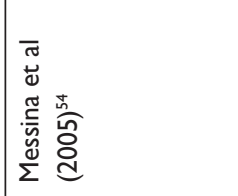 & 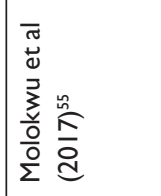 \\
\hline
\end{tabular}




\begin{tabular}{|c|c|c|c|c|c|c|c|c|}
\hline 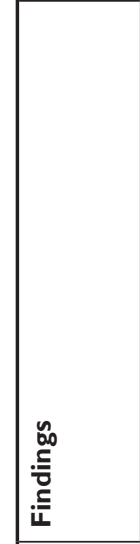 & 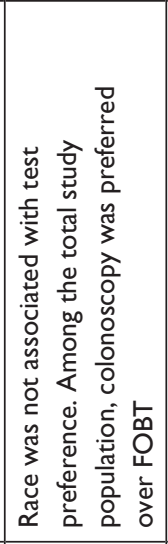 & 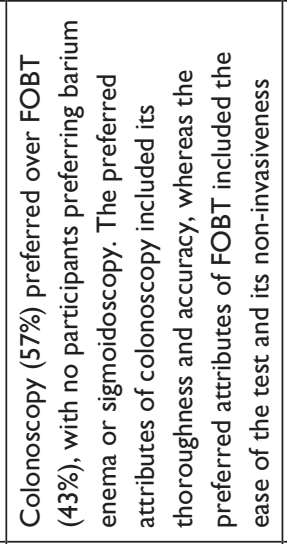 & 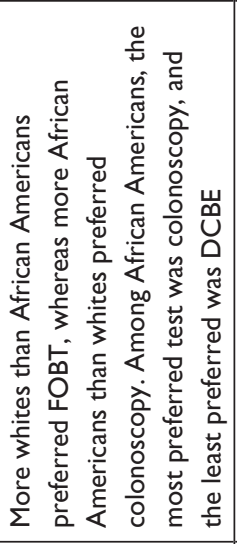 & 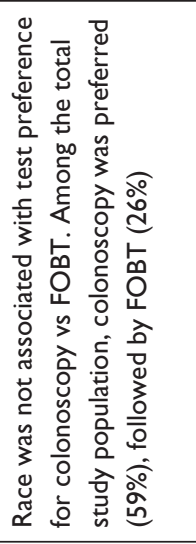 & 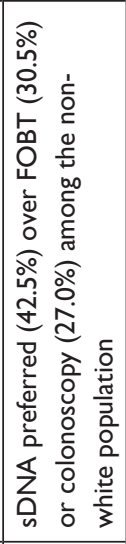 & 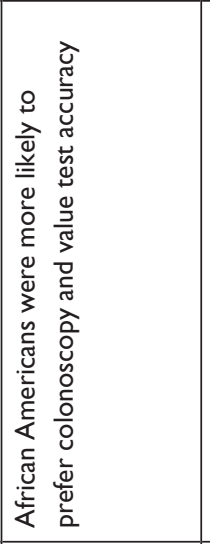 & 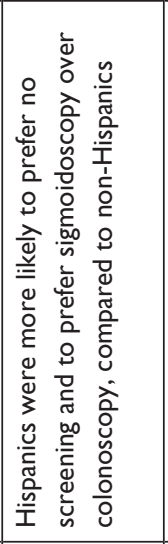 & 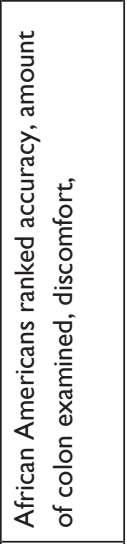 \\
\hline 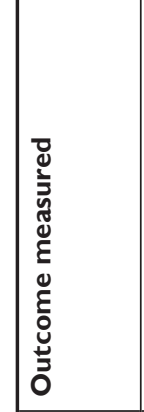 & 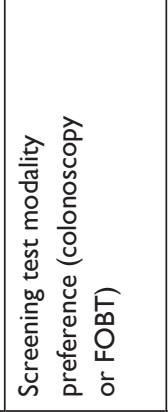 & 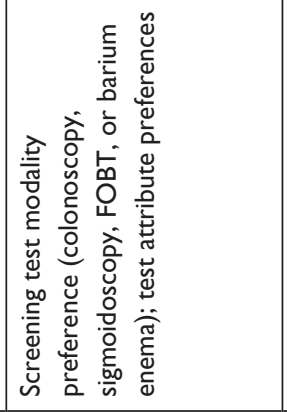 & 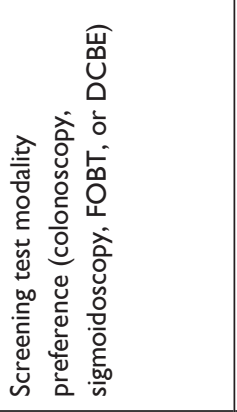 & 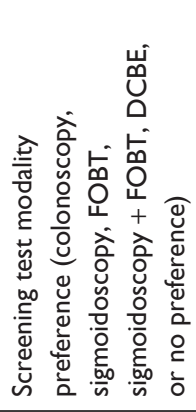 & 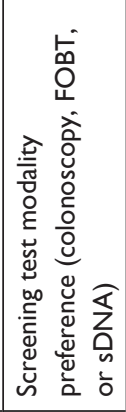 & 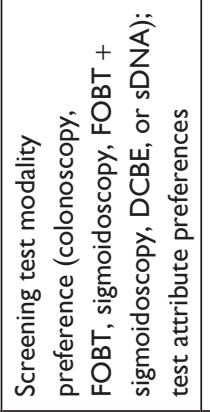 & 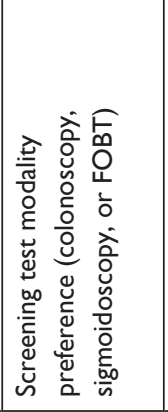 & 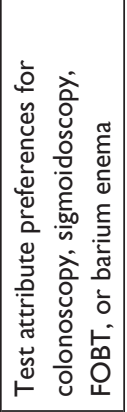 \\
\hline 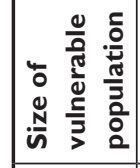 & నิ & 8 & in & $\frac{\infty}{\sigma}$ & $\stackrel{\substack{\infty \\
m}}{n}$ & $\approx$ & $\stackrel{\infty}{m}$ & $\stackrel{\infty}{\underline{0}}$ \\
\hline 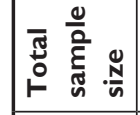 & 兽 & 8 & $\stackrel{m}{2}$ & : & $\stackrel{\substack{m \\
m}}{\stackrel{m}{m}}$ & $\stackrel{\tilde{N}}{\tilde{N}}$ & $\underline{\varrho}$ & $\stackrel{\infty}{\stackrel{\infty}{ }}$ \\
\hline 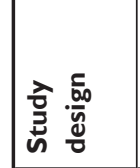 & $\begin{array}{l}\stackrel{\vec{े}}{\vec{s}} \\
\bar{s}\end{array}$ & 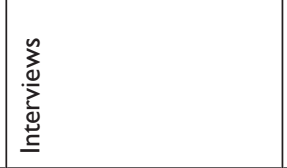 & 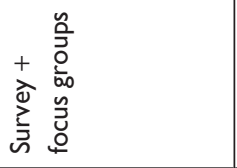 & $\underset{\mathscr{\complement}}{\mathfrak{u}}$ & 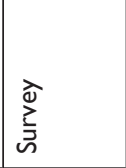 & 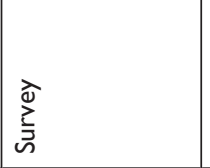 & 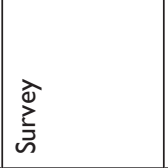 & 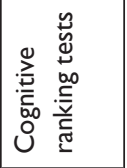 \\
\hline 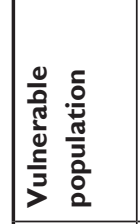 & 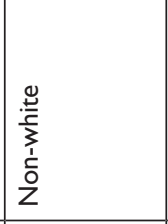 & 胥 & 虑 & 胥 & 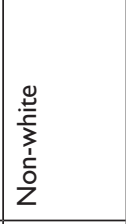 & 胥 & 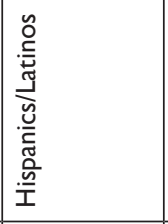 & 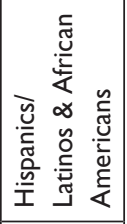 \\
\hline 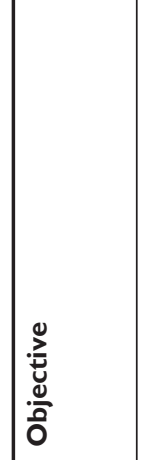 & 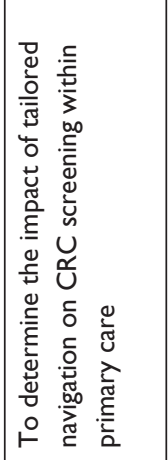 & 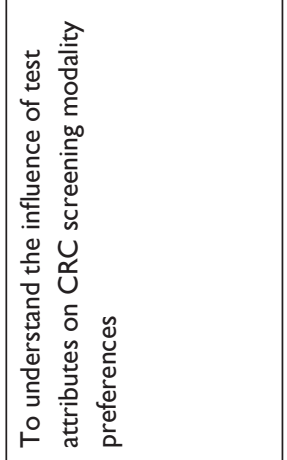 & 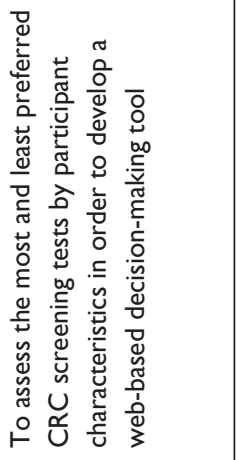 & 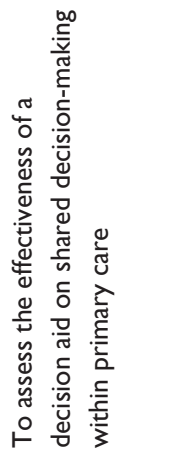 & 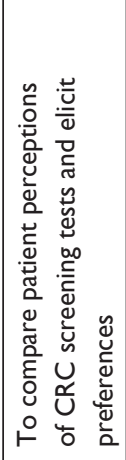 & 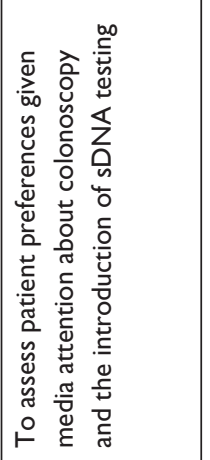 & 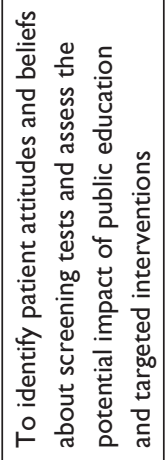 & 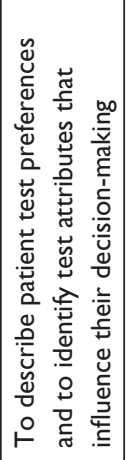 \\
\hline 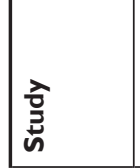 & 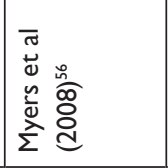 & 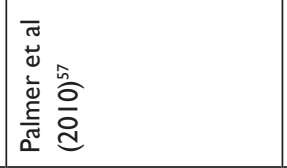 & 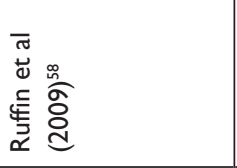 & 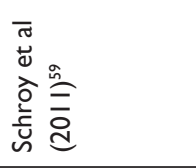 & 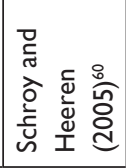 & 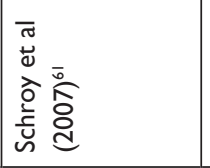 & 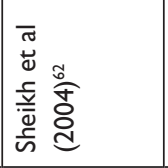 & 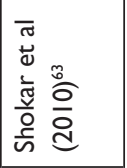 \\
\hline
\end{tabular}




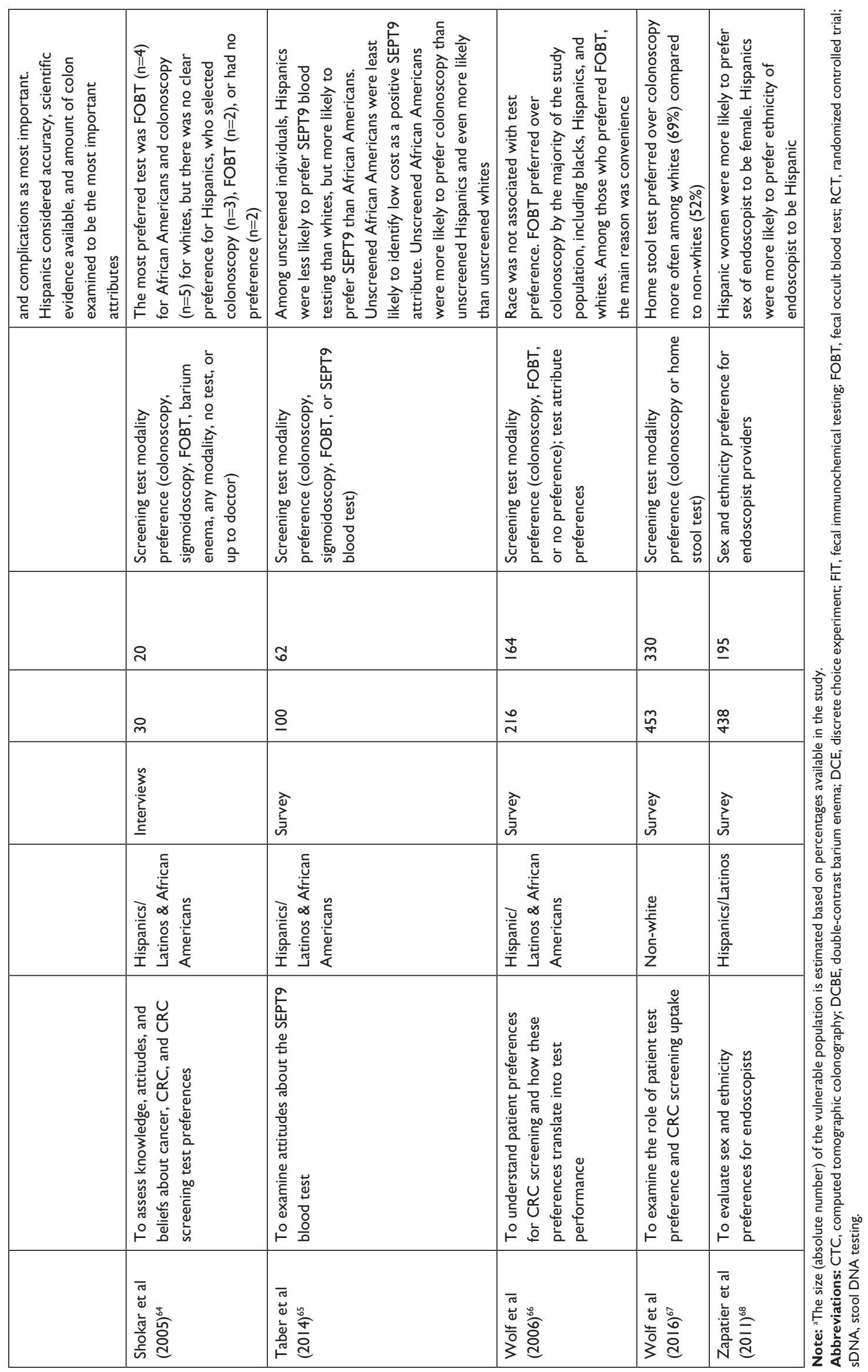


determined that study participants had the strongest desire to learn from an educational session, followed by the Korean media and print materials. ${ }^{52}$ In surveys with Hispanic/Latino patients, Ellison et al found that the preferred sources of information included providers, health brochures, television, and someone who speaks the same language. ${ }^{47}$ Therefore, there is evidence to suggest that ethnic minority patients look to cultural sources for resources related to preventive care.

\section{Decision-making process}

The two articles that studied decision-making preferences indicated the importance of shared decision-making for CRC screening among African Americans ${ }^{54}$ and Hispanics/ Latinos. ${ }^{55}$ For example, Molokwu et al found that over half of the Hispanic/Latino participants preferred a collaborative role, rather than a passive or active role, in the decisionmaking process. ${ }^{55}$ Similarly, Messina et al determined that African Americans, as compared to whites, would rather engage in shared decision-making than make CRC screening decisions independently. ${ }^{54}$

\section{Provider demographics}

Zapatier et al found that Hispanics overall exhibited a preference for the sex and ethnicity of endoscopist. ${ }^{68}$ In particular, Hispanic women prefer to have a female endoscopist and Hispanics regardless of sex prefer to have an endoscopist who is also Hispanic. ${ }^{68}$

\section{Low-income populations $(\mathrm{n}=8)$}

Eight articles reported CRC screening preferences among low-income populations (Table 3). ${ }^{69-76}$ Six of these studies were conducted in an international setting, ${ }^{69,70,72-75}$ and two were conducted domestically. ${ }^{71,76}$ Four studies reported monthly incomes, ${ }^{69,72,74,75}$ two studies reported annual incomes, ${ }^{70,76}$ one study used social grade as a proxy for socioeconomic status, ${ }^{73}$ and one study only included participants with an annual household income $<150 \%$ of the federal poverty level (FPL). ${ }^{71}$ Since the studies were conducted in different settings, most did not use a standardized threshold for defining low income, such as a percentage of the FPL. With the exception of Quick et al, ${ }^{71}$ we instead included studies that provided a subgroup analysis by income or social grade and focused on the results for the lowest income or social grade category.

Six of the eight studies focused on preference of FIT over colonoscopy, ${ }^{69,71,72,74-76}$ while the other two studies focused on $\mathrm{WTP}^{70}$ and preference for an expert recommendation. ${ }^{73}$ In the WTP study, Frew et al found that low-income patients had lower WTP values for CRC screening and higher-income patients had higher WTP values. ${ }^{70}$ Quick et al pointed to a possible relationship between lower income and colonoscopy preference. ${ }^{71}$ Yet, Wong et al showed that low-income participants were more likely to shift preference from colonoscopy to FIT after an educational session, ${ }^{75}$ and two other studies found higher income to be associated with colonoscopy preference. ${ }^{69,76}$ In contrast, Saengow et al and Wong et al did not find any association between income and preference, ${ }^{72,74}$ and Waller et al did not find an association between lower social grade and preference for expert recommendation. ${ }^{73}$

\section{Veterans $(n=6)$}

Six studies sampled patients exclusively from the US Veterans Health Administration, and all focused on modality preferences (Table 4). ${ }^{77-82}$ Four of these studies indicated that colonoscopy was the preferred modality among veterans. ${ }^{77-79,81}$ Rajapaksa et al found that the preference for computed tomographic colonography (CTC) vs colonoscopy was not significantly different. ${ }^{82}$ This study also found that racial/ethnic minorities among the veteran population were less likely to prefer CTC over colonoscopy. ${ }^{82}$ Moawad et al is the only study that suggested that colonoscopy is not the preferred modality among veterans; rather, in this study, veterans preferred CTC. ${ }^{80}$

\section{Rural $(n=2)$}

Two studies focused primarily on US rural populations' preferences for CRC screening (Table 5) ${ }^{83,84}$ Pham et al studied a rural population that consisted of predominantly Hispanics, but a subgroup analysis to see whether preference varied by race was not conducted. ${ }^{83}$ This study assessed delivery attributes of different fecal test options and found that participants prefer tests that use probes and vials, require a single stool sample, and provide clear, visual instructions. ${ }^{83}$

Pignone et al sampled from a rural setting and included a large percentage of uninsured and low-income individuals. ${ }^{84}$ This study assessed preferences for four screening program delivery attributes (testing options available, travel time, money received for completing screening, and out-of-pocket follow-up care costs). Pignone et al showed that screening costs and follow-up costs are more important factors in rural patients' preferences than travel time and specific test modality and that participants value having the option of fecal testing, rather than only being offered colonoscopy. ${ }^{84}$

\section{Discussion}

This review provides insight into the current literature regarding the CRC screening preferences of vulnerable populations. This information can be used to strengthen targeted 
Table 3 Characteristics of studies with low-income populations

\begin{tabular}{|c|c|c|c|c|c|c|}
\hline Study & Objective & $\begin{array}{l}\text { Study } \\
\text { design }\end{array}$ & $\begin{array}{l}\text { Total } \\
\text { sample } \\
\text { size }\end{array}$ & $\begin{array}{l}\text { Sample size } \\
\text { of vulnerable } \\
\text { population }\end{array}$ & Outcome measure & Findings \\
\hline $\begin{array}{l}\text { Cho et al } \\
(2017)^{69}\end{array}$ & $\begin{array}{l}\text { To investigate patient } \\
\text { preferences for CRC } \\
\text { screening modality for the } \\
\text { National Cancer Screening } \\
\text { Program }\end{array}$ & Survey & 396 & 216 & $\begin{array}{l}\text { Screening test modality } \\
\text { preference (colonoscopy } \\
\text { or FIT) }\end{array}$ & $\begin{array}{l}\text { Higher income was } \\
\text { associated with preference } \\
\text { for colonoscopy over FIT }\end{array}$ \\
\hline $\begin{array}{l}\text { Frew et al } \\
(2001)^{70}\end{array}$ & $\begin{array}{l}\text { To examine the willingness } \\
\text { to pay for two types of } \\
\text { CRC screening }\end{array}$ & Survey & 2,767 & $549^{a}$ & Willingness to pay & $\begin{array}{l}\text { Lower income was } \\
\text { associated with lower } \\
\text { willingness to pay for } \\
\text { CRC screening }\end{array}$ \\
\hline $\begin{array}{l}\text { Quick et al } \\
(20 \mid 3)^{71}\end{array}$ & $\begin{array}{l}\text { To examine the effect test- } \\
\text { specific barriers have on } \\
\text { CRC screening completion, } \\
\text { reasons for non-completion, } \\
\text { and patterns when } \\
\text { participants are allowed to } \\
\text { switch modalities }\end{array}$ & Survey & 418 & 418 & $\begin{array}{l}\text { Screening test modality } \\
\text { preference (colonoscopy } \\
\text { or FIT) }\end{array}$ & $\begin{array}{l}\text { Low-income population } \\
\text { preferred colonoscopy } \\
(60 \%) \text { over FIT ( } 40 \%) \text { at } \\
\text { baseline }\end{array}$ \\
\hline $\begin{array}{l}\text { Saengow et al } \\
(2015)^{72}\end{array}$ & $\begin{array}{l}\text { To estimate CRC screening } \\
\text { test preferences and } \\
\text { acceptance and the reasons } \\
\text { for decisions about whether } \\
\text { to screen }\end{array}$ & Interviews & 437 & 167 & $\begin{array}{l}\text { Screening test modality } \\
\text { preference (colonoscopy } \\
\text { or FIT) }\end{array}$ & $\begin{array}{l}\text { Lower income was not } \\
\text { associated with screening } \\
\text { modality preference }\end{array}$ \\
\hline $\begin{array}{l}\text { Waller et al } \\
(2012)^{73}\end{array}$ & $\begin{array}{l}\text { To assess public } \\
\text { preferences for a CRC } \\
\text { screening recommendation } \\
\text { within the United } \\
\text { Kingdom's National } \\
\text { Health Service Colorectal } \\
\text { (Bowel) Cancer Screening } \\
\text { Programme }\end{array}$ & Survey & 1,964 & 701 & $\begin{array}{l}\text { Preference for expert } \\
\text { recommendation (a strong } \\
\text { recommendation for } \\
\text { FOBT, a recommendation } \\
\text { plus advice to make an } \\
\text { individual choice, or no } \\
\text { recommendation but advice } \\
\text { to make an individual } \\
\text { decision) }\end{array}$ & $\begin{array}{l}\text { Lower social grade } \\
\text { was not associated } \\
\text { with recommendation } \\
\text { preference }\end{array}$ \\
\hline $\begin{array}{l}\text { Wong et al } \\
(2010)^{74}\end{array}$ & $\begin{array}{l}\text { To evaluate CRC screening } \\
\text { test preferences }\end{array}$ & Survey & 3,430 & 1,006 & $\begin{array}{l}\text { Screening test modality } \\
\text { preference (colonoscopy } \\
\text { or FIT) }\end{array}$ & $\begin{array}{l}\text { Monthly income was not } \\
\text { associated with modality } \\
\text { preference }\end{array}$ \\
\hline $\begin{array}{l}\text { Wong et al } \\
(2012)^{75}\end{array}$ & $\begin{array}{l}\text { To examine factors } \\
\text { influencing CRC screening } \\
\text { test choice and assess the } \\
\text { impact of an educational } \\
\text { session on this decision }\end{array}$ & Survey & 7,845 & 5,026 & $\begin{array}{l}\text { Screening test modality } \\
\text { preference (colonoscopy } \\
\text { or FIT) }\end{array}$ & $\begin{array}{l}\text { Lower income was } \\
\text { associated with greater } \\
\text { likelihood of changing } \\
\text { preference from } \\
\text { colonoscopy to FIT after } \\
\text { an educational intervention }\end{array}$ \\
\hline $\begin{array}{l}\text { Xu et al } \\
(20 \mid 5)^{76}\end{array}$ & $\begin{array}{l}\text { To assess patient } \\
\text { preferences for CRC } \\
\text { screening tests }\end{array}$ & Survey & 667 & 204 & $\begin{array}{l}\text { Screening test modality } \\
\text { preference (colonoscopy } \\
\text { or FIT) }\end{array}$ & $\begin{array}{l}\text { Higher household } \\
\text { income was associated } \\
\text { with greater likelihood } \\
\text { of preference for } \\
\text { colonoscopy }\end{array}$ \\
\hline
\end{tabular}

Note: ${ }^{a}$ The size (absolute number) of the vulnerable population is estimated based on percentages available in the study.

Abbreviations: CRC, colorectal cancer screening; FIT, fecal immunochemical testing.

interventions and policies seeking to address their relatively low screening rates. We found that there is not a single preferred CRC screening test across the identified vulnerable populations. Instead, these studies highlighted opportunities to better engage diverse patients in their preventive care decisions. These opportunities include facilitating a collaborative decision-making process regarding the type of modality used and eliciting individual patients' preferences about particular tests, for example, through a decision aid approach. The results demonstrate that efforts to promote CRC screening should address the wide range of testing modality options, since there is much variation in individual preferences. 
Table 4 Characteristics of studies with veteran populations

\begin{tabular}{|c|c|c|c|c|c|c|}
\hline Study & Objective & $\begin{array}{l}\text { Study } \\
\text { design }\end{array}$ & $\begin{array}{l}\text { Total } \\
\text { sample } \\
\text { size }\end{array}$ & $\begin{array}{l}\text { Sample size } \\
\text { of vulnerable } \\
\text { population }\end{array}$ & Outcome measure & Findings \\
\hline $\begin{array}{l}\text { Akerkar et al } \\
(2001)^{77}\end{array}$ & $\begin{array}{l}\text { To assess and } \\
\text { compare patients' } \\
\text { experiences with } \\
\text { virtual colonoscopy } \\
\text { and conventional } \\
\text { colonoscopy }\end{array}$ & Survey & 295 & 295 & $\begin{array}{l}\text { Screening test modality } \\
\text { preference (conventional } \\
\text { colonoscopy or virtual } \\
\text { colonoscopy); time } \\
\text { tradeoff }\end{array}$ & $\begin{array}{l}\text { Conventional colonoscopy } \\
\text { preferred over virtual } \\
\text { colonoscopy. Patients were } \\
\text { willing to wait an average } \\
\text { of } 4.9 \text { weeks to undergo } \\
\text { conventional colonoscopy } \\
\text { relative to virtual colonoscopy } \\
\text { from the time of discharge }\end{array}$ \\
\hline $\begin{array}{l}\text { Friedemann- } \\
\text { Sánchez et al } \\
(2007)^{78}\end{array}$ & $\begin{array}{l}\text { To explore } \\
\text { CRC screening } \\
\text { barriers, attitudes } \\
\text { and preferences } \\
\text { by gender }\end{array}$ & Focus groups & 70 & 70 & $\begin{array}{l}\text { Screening test modality } \\
\text { preference (colonoscopy, } \\
\text { sigmoidoscopy, FOBT, } \\
\text { or DCBE) }\end{array}$ & $\begin{array}{l}\text { Colonoscopy preferred over } \\
\text { other screening modalities and } \\
\text { perceived as the "complete" } \\
\text { screening examination }\end{array}$ \\
\hline $\begin{array}{l}\text { Imaeda et al } \\
(2010)^{79}\end{array}$ & $\begin{array}{l}\text { To elicit patient } \\
\text { values about CRC } \\
\text { screening test } \\
\text { attributes and the } \\
\text { influence on test } \\
\text { preferences }\end{array}$ & $\begin{array}{l}\text { Maximum } \\
\text { difference } \\
\text { scaling survey }\end{array}$ & 92 & 75 & $\begin{array}{l}\text { Screening test modality } \\
\text { preference (colonoscopy, } \\
\text { sigmoidoscopy, FOBT, } \\
\text { CTC, or colon capsule } \\
\text { endoscopy); test attribute } \\
\text { preferences }\end{array}$ & $\begin{array}{l}\text { Colonoscopy }(62 \%) \text { preferred } \\
\text { over other screening modalities. } \\
\text { Test sensitivity, risk of a } \\
\text { perforation, and potential } \\
\text { need for a follow-up test were } \\
\text { identified as the most important } \\
\text { attributes }\end{array}$ \\
\hline $\begin{array}{l}\text { Moawad et al } \\
(2010)^{80}\end{array}$ & $\begin{array}{l}\text { To assess patient } \\
\text { preferences for } \\
\text { screening modality }\end{array}$ & Survey & 250 & 250 & $\begin{array}{l}\text { Screening test modality } \\
\text { preference (colonoscopy } \\
\text { or CTC) }\end{array}$ & $\begin{array}{l}\text { CTC preferred over } \\
\text { colonoscopy by } 95 \% \text { of the study } \\
\text { population that completed both } \\
\text { colonoscopy and CTC }(n=54)\end{array}$ \\
\hline $\begin{array}{l}\text { Powell et al } \\
(2009)^{81}\end{array}$ & $\begin{array}{l}\text { To assess CRC } \\
\text { screening modality } \\
\text { preferences among } \\
\text { US veterans }\end{array}$ & Survey & 2,068 & 2,068 & $\begin{array}{l}\text { Screening test modality } \\
\text { preference (colonoscopy, } \\
\text { sigmoidoscopy, FOBT, } \\
\text { DCBE, or no screening) }\end{array}$ & $\begin{array}{l}\text { Colonoscopy }(37 \%) \text { and FOBT } \\
(29 \%) \text { preferred over other } \\
\text { screening modalities }\end{array}$ \\
\hline $\begin{array}{l}\text { Rajapaksa } \\
\text { et al }(2007)^{82}\end{array}$ & $\begin{array}{l}\text { To assess potential } \\
\text { differences in patient } \\
\text { experiences with and } \\
\text { preferences for CRC } \\
\text { screening modality }\end{array}$ & Survey & 272 & 272 & $\begin{array}{l}\text { Screening test modality } \\
\text { preference (optical } \\
\text { colonoscopy or CTC) }\end{array}$ & $\begin{array}{l}\text { No significant difference in } \\
\text { preference for CTC vs optical } \\
\text { colonoscopy in the total study } \\
\text { population. Racial and ethnic } \\
\text { minorities within the study } \\
\text { population were less likely } \\
\text { to prefer CRC over optical } \\
\text { colonoscopy relative to the } \\
\text { white participants }\end{array}$ \\
\hline
\end{tabular}

Abbreviations: CTC, computed tomographic colonography; DCBE, double-contrast barium enema; FOBT, fecal occult blood test.

A relatively large number of studies addressed CRC screening preferences among vulnerable patient populations $(\mathrm{n}=43)$; of these, seven studies were conducted in non-US developed countries. Most studies investigated the preferences of racial and ethnic minority groups, with few studies that focused on rural populations or immigrants. This is concerning, since the US comprises large swaths of rural areas and a growing immigrant population, making rural and immigrant groups important populations to consider. ${ }^{85,86}$ Many studies, especially those that focused on Hispanic/ Latinos, may have captured immigrant populations, but did not always record immigrant status. Likewise, while some studies included participants who were vulnerable in terms of insurance status (eg, Medicaid enrollees, the uninsured), these studies focused on preferences among a different population, such as racial or ethnic minorities or low-income populations. Notably, there have yet to be studies assessing preferences in two vulnerable populations identified in our inclusion criteria: individuals with disabilities and members of the LGBTQ community.

In terms of outcomes, most studies across vulnerable groups focused on preferences for test modality, most commonly contrasting colonoscopy and fecal testing. The high density of studies concentrating on modality points to the dearth of studies that measured test attribute, program features, how providers should approach CRC screening 
Table 5 Characteristics of studies with rural populations

\begin{tabular}{|l|l|l|l|l|l|l|}
\hline Study & Objective & $\begin{array}{l}\text { Study } \\
\text { design }\end{array}$ & $\begin{array}{l}\text { Total } \\
\text { sample } \\
\text { size }\end{array}$ & $\begin{array}{l}\text { Sample size } \\
\text { of vulnerable } \\
\text { population }\end{array}$ & Outcome measure & Findings \\
\hline $\begin{array}{l}\text { Pham et al } \\
(2017)^{83}\end{array}$ & $\begin{array}{l}\text { To assess patient } \\
\text { preferences for } \\
\text { FIT characteristics } \\
\text { in order to inform } \\
\text { regional FIT selection }\end{array}$ & $\begin{array}{l}\text { Survey }+ \\
\text { focus groups }\end{array}$ & 18 & 18 & $\begin{array}{l}\text { FIT-specific screening test } \\
\text { program delivery preferences }\end{array}$ & $\begin{array}{l}\text { FITs that required only one } \\
\text { sample, collected samples } \\
\text { using a probe and vial, and } \\
\text { included descriptive, visual } \\
\text { instructions were preferred }\end{array}$ \\
\hline $\begin{array}{l}\text { Pignone et al } \\
(2014)^{84}\end{array}$ & $\begin{array}{l}\text { To determine how } \\
\text { vulnerable individuals } \\
\text { value different aspects } \\
\text { of CRC screening } \\
\text { programs }\end{array}$ & DCE & 150 & 150 & $\begin{array}{l}\text { Preference for screening } \\
\text { program design/delivery, } \\
\text { including screening modality } \\
\text { options, travel time, money } \\
\text { paid for screening, and the } \\
\text { portion of the cost of follow- } \\
\text { up care paid out of pocket }\end{array}$ & $\begin{array}{l}\text { Coverage of follow-up costs } \\
\text { identified as more important } \\
\text { travel time }\end{array}$ \\
\hline
\end{tabular}

Abbreviations: DCE, discrete choice experiment; FIT, fecal immunochemical testing.

discussions, and other aspects of preference. There was relatively strong agreement regarding a preference for colonoscopy among veterans; however, results about test modality preferences were mixed among all other sub-populations. More focus on directly eliciting preferences for specific test attributes may help to clarify our understanding of which modality or screening program design would be ideal for specific sub-populations. Studies that investigated test attributes tended to find that accuracy, sensitivity, costs, frequency of test, convenience, and comfort were important. Accuracy and sensitivity were often associated with colonoscopy, while convenience was often associated with fecal testing. Regardless of the preferred modality, accuracy was the single most common attribute identified across sub-populations. In systematic reviews that focused on the general population, accuracy was also identified as an important attribute and no specific modality was dominantly preferred. ${ }^{20-22}$

The studies that measured preference for decision-making pointed to shared decision-making between patients and providers, highlighting a willingness by vulnerable patients to engage in productive and collaborative efforts to receive screening. This is paramount since consideration of patient preferences in terms of testing attributes can be combined with provider expertise to reach an optimal screening strategy. This review provides support for the use of decision aids among vulnerable sub-populations as a method of educating patients about their options and allowing patients to clarify their preferences regarding test features and screening modalities. ${ }^{43,87-89}$

This study includes several strengths. This is the first systematic review, to our knowledge, to address CRC screening preferences among vulnerable populations, compiling studies from more than a 20 -year timeframe. We also highlighted the relatively large number of studies focused on modality preferences and the limited research available on other important features of the decision-making process.

However, there were also a number of limitations. In order to gauge the scope of articles addressing vulnerable patients' CRC screening preferences, we included a broad range of study designs, focus populations and outcome measurement, so the studies are not all directly comparable. For example, given that a large proportion of the studies primarily addressing low-income patients were international, cultural contexts may have influenced the results, making it difficult to generalize to US sub-populations. There may also be additional populations that could be considered vulnerable in this context, such as patients who are illiterate or have low educational levels, not included in this review. In addition, the intersectionality of identities among the vulnerable populations made it difficult to elicit a specific preference for a singular categorization. As a result, we caution against making sweeping generalizations about the preferences of these sub-groups, due to the variety of factors that influence preferences for CRC screening in specific subpopulations. Instead, this review elicited trends and themes from the literature and can be used as a guide for planning and implementing CRC screening interventions that are well-aligned with patients' stated preferences, underlying barriers and facilitators to screening, and realities of local settings and contexts..$^{90}$

This systematic review highlights several opportunities for future research to ensure CRC screening programs better align with the preferences of vulnerable patients and ultimately to improve their CRC screening rates. First, more standardized methods to capture preferences, such as discrete choice experiments and conjoint analyses, may be 
needed to clarify tradeoffs, especially since a single modality preference was not found. Although many discrete choice experiments focused on CRC screening were conducted among the general population, few have focused on specific vulnerable sub-groups. Second, since multiple modalities are generally acceptable, it will be important to determine how frequently providers are offering multiple test options. Third, future research should consider the best approach to presenting screening test options to vulnerable patients in order to create a balance between providing patients with options that are consistent with their values and offer them flexibility but not providing an overwhelming number of options and features to consider. This is especially critical given that patients reported interest in a shared decisionmaking process, but it remains unclear how providers should initiate these discussions. Fourth, preference studies should be conducted among those groups for whom preferences have not yet been assessed, such as disabled individuals and LGBTQ individuals. Finally, assessment activities are needed to inform intervention design and create alignment between testing preferences and screening interventions.

\section{Conclusion}

Our systematic review of CRC screening preferences in vulnerable populations revealed substantial heterogeneity in outcomes measured, study design, and populations studied and demonstrated a wide spectrum of CRC screening preferences across different vulnerable populations. This review echoes the results of previous systematic reviews conducted on $\mathrm{CRC}$ screening preferences among the general population in that there is no specific test modality that is overwhelmingly supported by vulnerable populations; rather, having a choice between modalities may be preferred, especially in the context of shared decision-making, which vulnerable patients seem to value. All studies measuring patients' preferences for decision-making included in this review pointed to an engaged and shared decision-making between the patient and provider. In addition, screening test attributes such as accuracy, sensitivity, cost, and convenience are important features to consider. More studies that measure the various aspects of preference beyond test modalities alone are needed in the current literature.

To increase CRC screening overall, special attention must be paid to vulnerable populations that struggle with a lower screening uptake due to differential preferences and other reasons that may diverge from the general population (eg, ability to access and pay for follow-up care). The diverse findings reported in this review point to the increased value of decision aids to elicit individually how vulnerable patients weigh certain attributes against each other when making a screening decision. Improvements in CRC screening rates may be achieved through the alignment of vulnerable sub-populations' preferences with screening program delivery and provider practices, through decision aids or other approaches that seek to clarify and enhance patients' screening decisions.

\section{Acknowledgments}

This study was supported, in part, by Cooperative Agreement Number U48-DP005017 from the Centers for Disease Control and Prevention, Prevention Research Centers Program and the National Cancer Institute, as part of the Cancer Prevention and Control Research Network. The contents are solely the responsibility of the authors and do not necessarily represent the official view of the funders.

\section{Disclosure}

SBW received unrelated grant funding to her institution from Pfizer. The authors report no other conflicts of interest in this work.

\section{References}

1. Cancer Facts \& Figures 2017. Available from: https://www.cancer.org/ research/cancer-facts-statistics/all-cancer-facts-figures/cancer-factsfigures-2017.html. Accessed March 3, 2018.

2. Mariotto AB, Yabroff KR, Shao Y, Feuer EJ, Brown ML. Projections of the cost of cancer care in the United States: 2010-2020. J Natl Cancer Inst. 2011;103(2):117-128.

3. Whitlock EP, Lin JS, Liles E, Beil TL, Fu R. Screening for colorectal cancer: a targeted, updated systematic review for the U.S. Preventive Services Task Force. Ann Intern Med. 2008;149(9):638-658.

4. Maciosek MV, Solberg LI, Coffield AB, Edwards NM, Goodman MJ. Colorectal cancer screening: health impact and cost effectiveness. Am J Prev Med. 2006;31(1):80-89.

5. Shida H, Ban K, Matsumoto M, et al. Asymptomatic colorectal cancer detected by screening. Dis Colon Rectum. 1996;39(10):1130-1135.

6. U.S. Preventive Services Task Force. Screening for colorectal cancer: U.S. Preventive Services Task Force recommendation statement. Ann Intern Med. 2008;149(9):627-637.

7. Centers for Disease Control and Prevention (CDC). Vital signs: colorectal cancer screening test use-United States, 2012. MMWR Morb Mortal Wkly Rep. 2013;62(44):881-888.

8. Society AC. Colorectal Cancer Facts \& Figures 2017-2019. Atlanta, GA: American Cancer Society. 2017.

9. Cancer. Cancer| Healthy People; 2020. Available from: https://www. healthypeople.gov/2020/topics-objectives/topic/cancer/objectives. Accessed November 1, 2017.

10. Goel MS, Wee CC, Mccarthy EP, Davis RB, Ngo-Metzger Q, Phillips RS. Racial and ethnic disparities in cancer screening: the importance of foreign birth as a barrier to care. J Gen Intern Med. 2003;18(12): 1028-1035.

11. Jackson CS, Oman M, Patel AM, Vega KJ. Health disparities in colorectal cancer among racial and ethnic minorities in the United States. J Gastrointest Oncol. 2016;7(Suppl 1):S32-S43.

12. James TM, Greiner KA, Ellerbeck EF, Feng C, Ahluwalia JS. Disparities in colorectal cancer screening: a guideline-based analysis of adherence. Ethn Dis. 2006;16(1):228-233.

13. Decker KM, Singh H. Reducing inequities in colorectal cancer screening in North America. J Carcinog. 2014;13:12. 
14. Liss DT, Baker DW. Understanding current racial/ethnic disparities in colorectal cancer screening in the United States: the contribution of socioeconomic status and access to care. Am J Prev Med. 2014;46(3): 228-236.

15. Walsh JM, Posner SF, Perez-Stable EJ. Colon cancer screening in the ambulatory setting. Prev Med. 2002;35(3):209-218.

16. Kiviniemi MT, Hay JL, James AS, et al. Decision making about cancer screening: an assessment of the state of the science and a suggested research agenda from the ASPO Behavioral Oncology and Cancer Communication Special Interest Group. Cancer Epidemiol Biomarkers Prev. 2009;18(11):3133-3137.

17. Nguyen TT, Mcphee SJ. Patient-provider communication in cancer screening. J Gen Intern Med. 2003;18(5):402-403.

18. Pignone M, Bucholtz D, Harris R. Patient preferences for colon cancer screening. J Gen Intern Med. 1999;14(7):432-437.

19. Geiger TM, Ricciardi R. Screening options and recommendations for colorectal cancer. Clin Colon Rectal Surg. 2009;22(4): 209-217.

20. Wortley S, Wong G, Kieu A, Howard K. Assessing stated preferences for colorectal cancer screening: a critical systematic review of discrete choice experiments. Patient. 2014;7(3):271-282.

21. Ghanouni A, Smith SG, Halligan S, et al. Public preferences for colorectal cancer screening tests: a review of conjoint analysis studies. Expert Rev Med Devices. 2013;10(4):489-499.

22. Marshall D, Mcgregor SE, Currie G. Measuring preferences for colorectal cancer screening: what are the implications for moving forward? Patient. 2010;3(2):79-89.

23. Hegarty V, Burchett BM, Gold DT, Cohen HJ. Racial differences in use of cancer prevention services among older Americans. J Am Geriatr Soc. 2000;48(7):735-740.

24. Sambamoorthi U, Mcalpine DD, Racial MDD. Racial, ethnic, socioeconomic, and access disparities in the use of preventive services among women. Prev Med. 2003;37(5):475-484.

25. Elnicki DM, Morris DK, Shockcor WT. Patient-perceived barriers to preventive health care among indigent, rural Appalachian patients. Arch Intern Med. 1995;155(4):421-424.

26. Shi L, Lebrun LA, Zhu J, Tsai J. Cancer screening among racial/ethnic and insurance groups in the United States: a comparison of disparities in 2000 and 2008. J Health Care Poor Underserved. 2011;22(3): 945-961.

27. Solberg LI, Brekke ML, Kottke TE. Are physicians less likely to recommend preventive services to low-SES patients? Prev Med. 1997; 26(3):350-357.

28. Moher D, Liberati A, Tetzlaff J, Altman DG, PRISMA Group. Preferred reporting items for systematic reviews and meta-analyses: the PRISMA statement. PLoS Med. 2009;6(7):e1000097.

29. Riva JJ, Malik KM, Burnie SJ, Endicott AR, Busse JW. What is your research question? An introduction to the PICOT format for clinicians. J Can Chiropr Assoc. 2012;56(3):167-171.

30. National Quality Forum. A roadmap for promoting health equity and eliminating disparities: the four I's for health equity. Available from: http://www.qualityforum.org/Publications/2017/09/A_Roadmap_ for_Promoting_Health_Equity_and_Eliminating_Disparities_The_ Four_I_s_for_Health_Equity.aspx. Accessed April 5, 2018.

31. Fiscella K, Sanders MR. Racial and ethnic disparities in the quality of health care. Annu Rev Public Health. 2016;37:375-394.

32. Koh HK, Oppenheimer SC, Massin-Short SB, Emmons KM, Geller AC, Viswanath K. Translating research evidence into practice to reduce health disparities: a social determinants approach. Am J Public Health. 2010;100(Suppl 1):S72-S80.

33. Kondo K, Low A, Everson T. Health disparities in veterans: a map of the evidence. Med Care. 2017;55:S9-S15.

34. Krahn GL, Walker DK, Correa-de-Araujo R. Persons with disabilities as an unrecognized health disparity population. Am J Public Health. 2015; 105(Suppl 2):S198-S206.

35. Institute of Medicine (IOM). Crossing the Quality Chasm: A New Health System for the 21st Century. Washington, DC: National Academies Press; 2001.
36. Institute of Medicine (IOM). Unequal Treatment: Confronting Racial and Ethnic Disparities in Health Care. Washington, DC: National Academies Press; 2003.

37. Agency for Healthcare Research and Quality (AHRQ). 2015 National Healthcare Quality and Disparities Report and 5th Anniversary Update on the National Quality Strategy. Rockville, MD: AHRQ; 2016. Available from: http://www.ahrq.gov/research/findings/nhqrdr/nhqdr15/ index.html. Accessed April 5, 2018.

38. Agency for Healthcare Research and Quality (AHRQ). 2016 National Healthcare Quality and Disparities Report. Rockville, MD: AHRQ. 2017.

39. HHS, Office of Minority Health. National Stakeholder Strategy for Achieving Health Equity. Washington, DC: HHS; 2016. Available from: http://www.minorityhealth.hhs.gov/npa/files/Plans/NSS/ NSS_05_Section1.pdf. Accessed 20 August 2018.

40. HHS. LGBT Health and Well-Being. Available from: https://www. hhs.gov/programs/topic-sites/lgbt/reports/health-objectives-2015.html. Accessed April 5, 2018.

41. Levin B, Bond JH. Colorectal cancer screening: recommendations of the U.S. Preventive Services Task Force. American Gastroenterological Association. Gastroenterology. 1996;111(5):1381-1384.

42. Abola MV, Fennimore TF, Chen MM, et al. Stool DNA-based versus colonoscopy-based colorectal cancer screening: patient perceptions and preferences. Fam Med Community Health. 2015;3(3):2-8.

43. Brenner AT, Hoffman R, Mcwilliams A, et al. Colorectal cancer screening in vulnerable patients: promoting informed and shared decisions. Am J Prev Med. 2016;51(4):454-462.

44. Calderwood AH, Wasan SK, Heeren TC, Schroy PC. Patient and provider preferences for colorectal cancer screening: how does CT colonography compare to other modalities? Int J Canc Prev. 2011;4(4):307-338.

45. Chablani SV, Cohen N, White D, Itzkowitz SH, Duhamel K, Jandorf L. Colorectal cancer screening preferences among Black and Latino primary care patients. J Immigr Minor Health. 2017;19(5):1100-1108.

46. Debourcy AC, Lichtenberger S, Felton S, Butterfield KT, Ahnen DJ, Denberg TD. Community-based preferences for stool cards versus colonoscopy in colorectal cancer screening. J Gen Intern Med. 2008;23(2): 169-174.

47. Ellison J, Jandorf L, Duhamel K. Colonoscopy screening information preferences among urban Hispanics. J Immigr Minor Health. 2011; 13(5):963-966.

48. Greiner KA, Born W, Nollen N, Ahluwalia JS. Knowledge and perceptions of colorectal cancer screening among urban African Americans. J Gen Intern Med. 2005;20(11):977-983.

49. Harden E, Moore A, Melvin C. Exploring perceptions of colorectal cancer and fecal immunochemical testing among African Americans in a North Carolina community. Prev Chronic Dis. 2011;8(6):A134.

50. Hawley ST, Mcqueen A, Bartholomew LK, et al. Preferences for colorectal cancer screening tests and screening test use in a large multispecialty primary care practice. Cancer. 2012;118(10):2726-2734.

51. Hawley ST, Volk RJ, Krishnamurthy P, Jibaja-Weiss M, Vernon SW, Kneuper S. Preferences for colorectal cancer screening among racially/ ethnically diverse primary care patients. Med Care. 2008;46(9 Suppl 1): S10-S16.

52. Jo AM, Maxwell AE, Wong WK, Bastani R. Colorectal cancer screening among underserved Korean Americans in Los Angeles County. J Immigr Minor Health. 2008;10(2):119-126.

53. Martens CE, Crutchfield TM, Laping JL, et al. Why wait until our community gets cancer?: exploring CRC screening barriers and facilitators in the Spanish-speaking community in North Carolina. J Cancer Educ. 2016;31(4):652-659.

54. Messina CR, Lane DS, Grimson R. Colorectal cancer screening attitudes and practices preferences for decision making. Am J Prev Med. 2005;28(5):439-446.

55. Molokwu JC, Penaranda E, Shokar N. Decision-making preferences among older Hispanics participating in a colorectal cancer (CRC) screening program. J Community Health. 2017;42(5):1027-1034.

56. Myers RE, Hyslop T, Sifri R, et al. Tailored navigation in colorectal cancer screening. Med Care. 2008;46(9 Suppl 1):S123-S131. 
57. Palmer RC, Midgette LA, Mullan ID. Colorectal cancer screening preferences among African Americans: which screening test is preferred? J Cancer Educ. 2010;25(4):577-581.

58. Ruffin MT, Creswell JW, Jimbo M, Fetters MD. Factors influencing choices for colorectal cancer screening among previously unscreened African and Caucasian Americans: findings from a triangulation mixed methods investigation. J Community Health. 2009;34(2):79-89.

59. Schroy PC, Emmons K, Peters E, et al. The impact of a novel computer-based decision aid on shared decision making for colorectal cancer screening: a randomized trial. Med Decis Making. 2011;31(1): 93-107.

60. Schroy PC, Heeren TC. Patient perceptions of stool-based DNA testing for colorectal cancer screening. Am J Prev Med. 2005;28(2):208-214.

61. Schroy PC, Lal S, Glick JT, Robinson PA, Zamor P, Heeren TC. Patient preferences for colorectal cancer screening: how does stool DNA testing fare? Am J Manag Care. 2007;13(7):393-400.

62. Sheikh RA, Kapre S, Calof OM, Ward C, Raina A. Screening preferences for colorectal cancer: a patient demographic study. South Med J. 2004;97(3):224-230.

63. Shokar NK, Carlson CA, Weller SC. Informed decision making changes test preferences for colorectal cancer screening in a diverse population. Ann Fam Med. 2010;8(2):141-150.

64. Shokar NK, Vernon SW, Weller SC. Cancer and colorectal cancer: knowledge, beliefs, and screening preferences of a diverse patient population. Fam Med. 2005;37(5):341-347.

65. Taber JM, Aspinwall LG, Heichman KA, Kinney AY. Preferences for blood-based colon cancer screening differ by race/ethnicity. Am J Health Behav. 2014;38(3):351-361.

66. Wolf RL, Basch CE, Brouse CH, Shmukler C, Shea S. Patient preferences and adherence to colorectal cancer screening in an urban population. Am J Public Health. 2006;96(5):809-811.

67. Wolf RL, Basch CE, Zybert P, et al. Patient test preference for colorectal cancer screening and screening uptake in an insured urban minority population. J Community Health. 2016;41(3):502-508.

68. Zapatier JA, Kumar AR, Perez A, Guevara R, Schneider A. Preferences for ethnicity and sex of endoscopists in a Hispanic population in the United States. Gastrointest Endosc. 2011;73(1):89-97.

69. Cho YH, Kim DH, Cha JM, et al. Patients' preferences for primary colorectal cancer screening: a survey of the national colorectal cancer screening program in Korea. Gut Liver. 2017;11(6):821-827.

70. Frew E, Wolstenholme JL, Whynes DK. Willingness-to-pay for colorectal cancer screening. Eur J Cancer. 2001;37(14):1746-1751.

71. Quick BW, Hester CM, Young KL, Greiner KA. Self-reported barriers to colorectal cancer screening in a racially diverse, low-income study population. J Community Health. 2013;38(2):285-292.

72. Saengow U, Chongsuwiwatvong V, Geater A, Birch S. Preferences and acceptance of colorectal cancer screening in Thailand. Asian Pac J Cancer Prev. 2015;16(6):2269-2276.

73. Waller J, Macedo A, von Wagner C, et al. Communication about colorectal cancer screening in Britain: public preferences for an expert recommendation. Br J Cancer. 2012;107(12):1938-1943.

74. Wong MC, Tsoi KK, Ng SS, et al. A comparison of the acceptance of immunochemical faecal occult blood test and colonoscopy in colorectal cancer screening: a prospective study among Chinese. Aliment Pharmacol Ther. 2010;32(1):74-82.
75. Wong MC, John GK, Hirai HW, et al. Changes in the choice of colorectal cancer screening tests in primary care settings from 7,845 prospectively collected surveys. Cancer Causes Control. 2012;23(9):1541-1548.

76. Xu Y, Levy BT, Daly JM, Bergus GR, Dunkelberg JC. Comparison of patient preferences for fecal immunochemical test or colonoscopy using the analytic hierarchy process. BMC Health Serv Res. 2015;15:175.

77. Akerkar GA, Yee J, Hung R, Mcquaid K. Patient experience and preferences toward colon cancer screening: a comparison of virtual colonoscopy and conventional colonoscopy. Gastrointest Endosc. 2001; 54(3):310-315.

78. Friedemann-Sánchez G, Griffin JM, Partin MR. Gender differences in colorectal cancer screening barriers and information needs. Health Expect. 2007;10(2):148-160.

79. Imaeda A, Bender D, Fraenkel L. What is most important to patients when deciding about colorectal screening? J Gen Intern Med. 2010; 25(7):688-693.

80. Moawad FJ, Maydonovitch CL, Cullen PA, Barlow DS, Jenson DW, Cash BD. CT colonography may improve colorectal cancer screening compliance. AJR. 2010;195(5):1118-1123.

81. Powell AA, Burgess DJ, Vernon SW, et al. Colorectal cancer screening mode preferences among US veterans. Prev Med. 2009;49(5): 442-448.

82. Rajapaksa RC, Macari M, Bini EJ. Racial/ethnic differences in patient experiences with and preferences for computed tomography colonography and optical colonoscopy. Clin Gastroenterol Hepatol. 2007; 5(11):1306-1312.

83. Pham R, Cross S, Fernandez B, et al. "Finding the right FIT": rural patient preferences for fecal immunochemical test (FIT) characteristics. J Am Board Fam Med. 2017;30(5):632-644.

84. Pignone MP, Crutchfield TM, Brown PM, et al. Using a discrete choice experiment to inform the design of programs to promote colon cancer screening for vulnerable populations in North Carolina. BMC Health Serv Res. 2014;14:611.

85. Phillips CD, Mcleroy KR. Health in rural America: remembering the importance of place. Am J Public Health. 2004;94(10):1661-1663.

86. López G, Bialik K. Key findings about U.S. immigrants. Pew Research Center. Available from: http://www.pewresearch.org/facttank/2017/05/03/key-findings-about-u-s-immigrants/. Accessed April 1, 2018 .

87. Hoffman AS, Lowenstein LM, Kamath GR, et al. An entertainmenteducation colorectal cancer screening decision aid for African American patients: a randomized controlled trial. Cancer. 2017; 123(8):1401-1408

88. Reuland DS, Brenner AT, Hoffman R, et al. Effect of combined patient decision aid and patient navigation vs usual care for colorectal cancer screening in a vulnerable patient population: a randomized clinical trial. JAMA Intern Med. 2017;177(7):967-974.

89. Volk RJ, Linder SK, Lopez-Olivo MA, et al. Patient decision aids for colorectal cancer screening: a systematic review and meta-analysis. Am J Prev Med. 2016;51(5):779-791.

90. Wheeler SB, Davis MM. "Taking the bull by the horns": four principles to align public health, primary care, and community efforts to improve rural cancer control. J Rural Health. 2017;33(4):345-349.
Patient Preference and Adherence

\section{Publish your work in this journal}

Patient Preference and Adherence is an international, peer-reviewed, open access journal that focuses on the growing importance of patient preference and adherence throughout the therapeutic continuum. Patient satisfaction, acceptability, quality of life, compliance, persistence and their role in developing new therapeutic modalities and compounds to optimize

\section{Dovepress}

clinical outcomes for existing disease states are major areas of interest for the journal. This journal has been accepted for indexing on PubMed Central. The manuscript management system is completely online and includes a very quick and fair peer-review system, which is all easy to use. Visit http://www dovepress.com/testimonials.php to read real quotes from published authors. 